quality of life of patients were assessed using the 43-point NIH CHRONIC PROSTATITIS SYMPTOM INDEX and a global response assessment over 2 years of follow-up.

Of the 293 patients who completed followup, 134 improved by 6 points or more on the NIH Chronic Prostatitis Symptom Index, while 20 worsened by 6 points. The mean improvement was 5 points; most of this occurred in the first 3 months of follow-up, however, and might be caused by intervention effects and regression to the mean. On the global response assessment, 136/445 patients reported a marked or moderate improvement, while 37 patients felt their condition had worsened. The 92 patients lost to follow-up tended to be younger, of lower income and with a lower level of education; they also tended to have the most severe symptoms.

At present, there is no explanation for the variation in patient response or for the dramatic fluctuation in symptoms reported by some patients with CP/CPPS. In an editorial comment, W Bushman recommends looking more closely at the causes of spontaneous remission, given the difficulty in developing effective treatments for CP/CPPS. He cautions that appropriate placebo controls are required in treatment studies for CP/CPPS, because of the large improvements seen in some patients.

Jim Casey

Original article Propert KJ et al. (2006) A prospective study of symptoms and quality of life in men with chronic prostatitis/chronic pelvic pain syndrome: The National Institutes of Health Chronic Prostatitis Cohort Study. J Urol 175: 619-623

\section{Role of basal acetylcholine release in bladder function}

Acetylcholine is the main neurotransmitter involved in bladder contraction. Immunohistochemical staining of choline acetyltransferase has revealed the presence of a non-neuronal cholinergic system widely distributed in human tissue. Researchers in Japan have, therefore, evaluated the non-neuronal cholinergic system in the human bladder. They used organs removed from patients with bladder cancer.

Electrical stimulation of bladder strips caused acetylcholine release that could be inhibited by tetrodotoxin, suggesting the release was neuronal in origin. In addition, however, a basal level of acetylcholine release was observed in unstimulated bladder tissue; this release was not tetrodotoxin-sensitive, suggesting that it did not originate from neurons. Stretching of bladder strips led to an increase in the level of basal acetylcholine release; the increase was much higher in bladder strips retaining their urothelium than in strips from which the urothelium had been removed. The level of stretch-induced acetylcholine release correlated positively with the age of the patient, as did the level of immunohistochemical staining for choline acetyltransferase.

These findings indicate that non-neuronal acetylcholine release occurs in the human bladder. The authors suggest that shear stress on the urothelium during bladder filling might be one of the release mechanisms, and speculate that non-neuronal acetylcholine release might contribute to the symptoms of overactive bladder in some patients. The precise role and mechanism of action of basal acetylcholine release in regulating bladder function, however, remain to be determined.

\section{Jim Casey}

Original article Yoshida M et al. (2006) Non-neuronal cholinergic system in human bladder urothelium. Urology 67: 425-430

\section{Saw palmetto might be no better than placebo for treating lower urinary tract symptoms}

In 2001, a systematic review of 21 short-term trials concluded that saw palmetto induced mild-to-moderate improvements in lower urinary tract symptoms (LUTS). Today, saw palmetto is used to treat LUTS in over 2 million US men. New findings from Bent et al. cast doubt on the herb's efficacy, however; their year-long study found no difference in LUTS between men who received saw palmetto capsules and those who received an identical-appearing placebo.

Bent et al. enrolled 225 men aged over 49 years with moderate-to-severe LUTS caused by benign prostatic hyperplasia, scored according to the american urological association sympt omindex (auasi), and a peak urinary flow rate of between $4 \mathrm{ml} / \mathrm{s}$ and $15 \mathrm{ml} / \mathrm{s}$. The men were randomly allocated to either $160 \mathrm{mg}$ saw
GLOSSARY

NIH CHRONIC

PROSTATITIS SYMPTOM INDEX

A nine-question validated symptom questionnaire with three domains (pain, urinary function, quality of life) that quantifies the degree of disability associated with chronic pelvic pain

AMERICAN UROLOGICAL ASSOCIATION SYMPTOM INDEX (AUASI)

$A$ validated seven-item questionnaire that scores lower-urinary-tract symptoms according to severity; scores of $0-7$, $8-19$, and $20-35$ correspond to mild, moderate and severe symptoms 